\title{
Accounting for the Effect of Rock Mass Anisotropy in Stress Measurements
}

\author{
P.M. Dight Coffey Mining Pty Ltd, Australia \\ A.V. Dyskin School of Civil and Resource Engineering, University of Western Australia, Australia
}

\begin{abstract}
Safety and efficiency of mining operations depend on the quality of design of mining excavations and sequence. A crucial ingredient in mining design is the adequate information of both the original in situ stress and the stress disturbance caused by mining. The main method of stress determination - the overcoring has a number of drawbacks. 1. It requires an open access to the location of the measurements, which is especially difficult if the stress is to be measured ahead of deep underground excavation. 2. It presumes the rock to be isotropic, while in many cases the rock anisotropy is significant. Subsequently, the isotropic stress reconstruction procedure of the overcoring method can give erroneous stress magnitudes and directions (e.g. Amadei and Goodman, 1982). The rock memory methods - DRA and the Kaiser effect are free from the influence of anisotropy. Moreover the DRA technique can, in principle, provide as a by-product a sufficient number of measurements to reconstruct the full anisotropic tensor of moduli, or compliances sufficient to characterise rock anisotropy, introduce corrections to the overcoring stress reconstruction procedure and quantify the effect of rock anisotropy on the stress concentrations induced by the excavation. In this paper, we present the procedure for the anisotropy characterisation based on DRA and analyse the sensitivity of the procedure and the effects of the rock anisotropy on stress measurements.
\end{abstract}

\section{Introduction}

There have been many attempts to identify the in situ stress from diamond drill core since J. Kaiser (1950, 1953) identified that materials have a stress memory even when tested well below their maximum strength in the "elastic" range. Kaiser discovered that acoustic emission (AE) could determine the previous maximum stress that the sample had experienced through the onset of a significant amount of noise/sound. The effect has become known as the Kaiser Effect (KE). The phenomenon has been confirmed in many laboratory tests (e.g. Goodman, 1963; Kurita and Fujii, 1979 (see also Lavrov, 2002). The effect has also been shown to measure the maximum previous stress measured in core recovered from diamond drilling (e.g. Villaescusa et al., 2003). The ability to obtain a measure of the stress field from core is clearly an incentive given the cost of alternate in situ techniques, such as Hydraulic fracture (HF), Hollow Inclusion cell (HI cell, CSIRO, NZ), borehole slotter, etc. This is even more important given that stress rotation/ local issues (lithology changes, faulting, etc) will impact on any point sample, so the need to get statistical samples. Here the HF technique and borehole slotter have a superior ability to obtain statistically significant numbers of samples than the HI technique. However, the assumptions in the former technique limit its usefulness and the latter technique has not been widely accepted. Yamamoto et al. (1990) published a comprehensive paper outlining the deformation rate analysis (DRA) technique for measuring KE. The approach examines the inelastic strain between successive load cycles in a uniaxial test. Simply, this states that the previous stress level in a sample can be detected by determining the strain difference between two successive cycles, numbered $i$ and $j$, of loading (known as the inelastic strain). If the response was elastic the difference would be zero. In mathematical form it is expressed as:

$$
\Delta e_{i, j}=e_{i}(\sigma)-e_{j}(\sigma), \quad j>i
$$

Yamamoto used the method to examine the maximum previous stress that the material had been tested to as per the AE method (Yamamoto et al. 1991, Yamamoto 1995). Since that time there has been some recent work where DRA had been used to examine the results of laboratory testing (Barr et al., 1999; Seto and Villaescusa 1999; Meyer et al., 2002). Meyer showed that DRA could provide information on each load step 
and hence the stress history could be understood from the test results. Using Meyer's concept, Dight (2006) showed that the current stress acting in the axis of the borehole could be determined from a DRA test, in addition to each load step and the previous maximum stress that the sample had experienced. This test was compared to the testing undertaken by WASM (2003 Rio Tinto - personal communication) using the AE technique. It was apparent from the initial tests undertaken by Dight that the maximum previous stress experienced by the core, as measured by DRA and KE, could have occurred during sampling. This effect is seen in HI testing and was also observed by Leeman (1965a, b, c) when first developing the Doorstopper technique. It can also be shown numerically (Dight, 2006; Lim et al., 2006) where a stress bubble appears ahead of coring. From testing in the laboratory and numerically, the influence can result in a stress change of between 1.2 and 2.6 times the maximum principal stress, plus it induces a rotation of the stress tensor associated with the rock fabric and intact properties. There could be other reasons as well, which are not yet well understood. Both DRA and AE are independent of the rock anisotropy.

Hakala et al. (2007) summarised the current status of in situ stress measurement in anisotropic rocks. This paper referenced the pioneering work of Amadei (1996) and reinforced the concerns that with an elastic anisotropy ratio of 1.5, an error of as much of $33 \%$ can be introduced into calculating the magnitudes of principal stress. Amadei (1983 ) showed that ratios of 1.14 to 1.33 will have a definite effect on the interpreted in situ state of stress, and presumably the orientation of the principal stress if the results are interpreted using a linear elastic isotropic solution. This was at variance to the views held by Worotnicki (1993) however it has yet to be properly evaluated.

Amadei (1983) produced the equations for analysing the stress results from a HI cell in an anisotropic medium, however to date being able to measure the anisotropy for use in the analyses has been difficult, unless the orientation of the anisotropy is known a priori.

Rock anisotropy can be attributed to fabric (rocks of sedimentary origin, which may have also been metamorphosed, and volcanic rocks, such as basalts and tuffs) and is made more complicated in particular near mineralised systems.

A consequence of the DRA technique is that the moduli and Poisson's ratio can be measured for each test direction. Not only is this a test of sample preparation compliance, it also provides a rich source of information on the rock anisotropy.

\section{Site characterisation and laboratory testing}

The geological setting for the case study described in this paper is characterised by meta sedimentary turbidite of Ordovician age. The sequence has been metamorphosed to greenschist facies and is extremely deformed.

Both DRA and HI testing have been undertaken for this site, with DRA testing undertaken on the overcore from the HI test. The testing was undertaken by two separate groups and the results were presented as a blind result. Both groups identified that anisotropy was an issue; however, it was not clear how this could be taken into account and whether the actual stress tensor results would vary in the case of the HI results if the anisotropy was taken into account. At the time it was not appreciated that the transverse isotropic parameters could be readily determined from the DRA test.

The tests were conducted at a depth below surface of $250 \mathrm{~m}$. The orientation of the foliation was $72^{\circ} / 096^{\circ}$ and the hole orientation was $-5^{\circ} / 047^{\circ}$ ( $5^{\circ}$ up). The testing of the subcores for DRA showed moduli ranging from $15 \mathrm{GPa}$ to $71 \mathrm{GPa}$ with a mean of $48 \mathrm{GPa}$, and a standard deviation of $16 \mathrm{GPa}$. Poisson's ratio varied from 0.14 to 0.46 with a mean of 0.32 and a standard deviation of 0.11 . Similar results were also noted from testing of representative samples from the HI testing, where the mean modulus result was $45 \mathrm{GPa}$ and the mean Poisson's ratio was 0.3. The Young's modulus varied from $13 \mathrm{GPa}$ to $60 \mathrm{GPa}$ for the circumferential gauges and Poisson's ratio varied from $0.3 \pm 0.114$. Figure 1 is a reconstruction of the moduli obtained from sub-sampling parallel to the direction of the circumferential strain gauges in the HI cell, which highlights the apparent variability of the rock modulus around the axis of the cell.

For the DRA results, they refer to moduli in particular directions corresponding to the orientations of the subcores. In order to understand the degree of anisotropy and analyse its influence, the moduli in the 
reference of a common (laboratory) co-ordinate frame should be determined. This will be done in the following section.

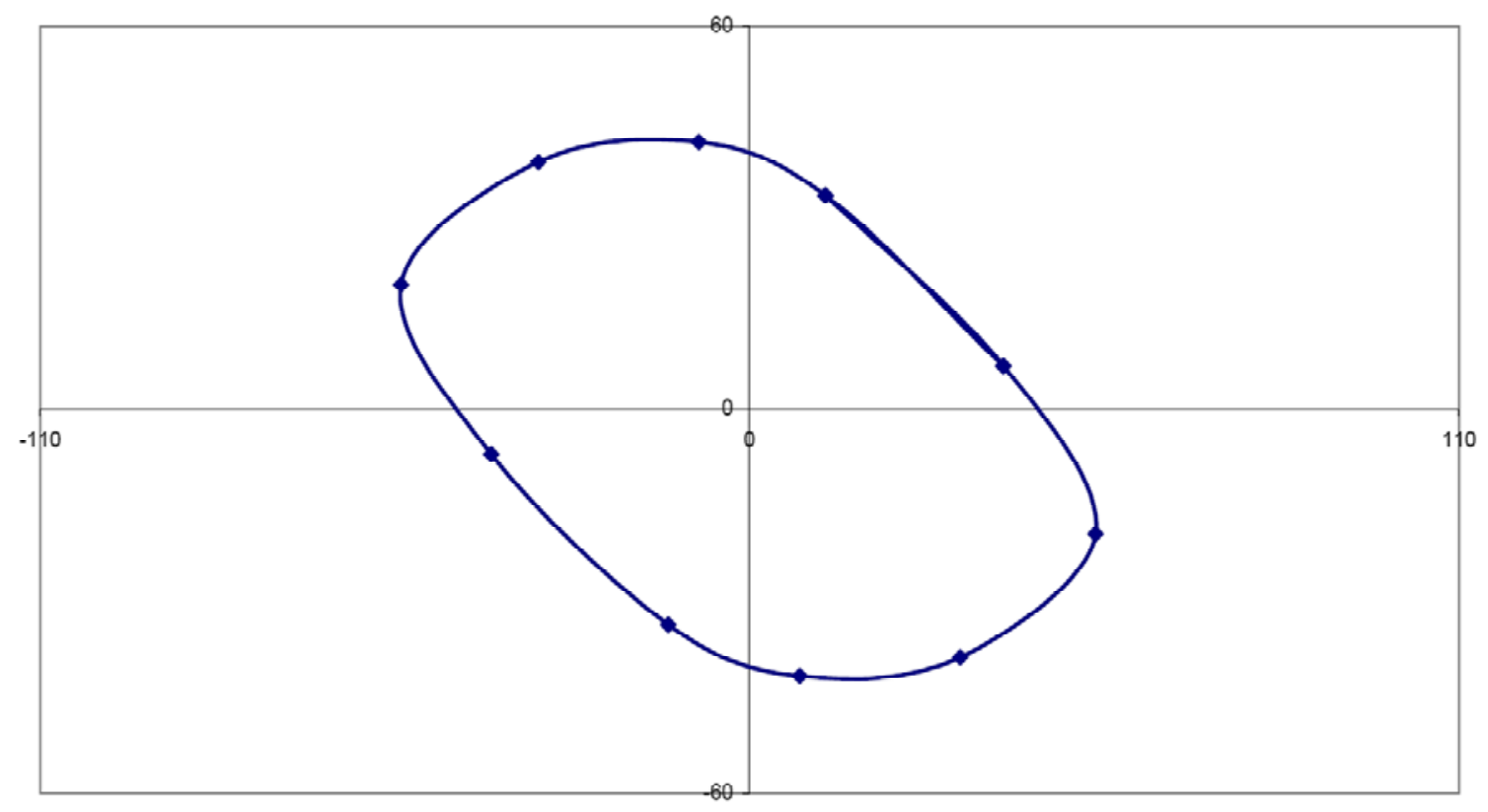

Figure 1 Moduli in axial directions of subcores inferred from the results of tests. The subcores were drilled in the directions of the circumferential gauges looking into the rock

\section{The procedure of reconstruction of anisotropic moduli}

\subsection{Characterisation of data available}

For the purpose of moduli reconstruction, the results of the laboratory tests on uniaxial compressive loading of subcores can be interpreted in the form of average axial and lateral strains measured in response to the axial load. We relate a subcore $n(n=1, \ldots, N$, where $N$ is the total number of subcores tested) to a local Cartesian co-ordinate set $\left(x^{(n)}, y^{(n)}, z^{(n)}\right)$ whose $z^{(n)}$ axis is directed along the axis of the subcore and $x^{(n)}$ axis is directed arbitrarily as the subcore orientation with respect to its axis was not marked. Subsequently, only two local compliances, $S_{\text {axial }}^{(n)}, \quad S_{\text {lateral }}^{(n)}$ can be determined corresponding to:

$$
\varepsilon_{z}^{(n)}=S_{\text {axial }}^{(n)} \sigma_{z}^{(n)}, \quad\left(\varepsilon_{x}^{(n)}+\varepsilon_{y}^{(n)}\right) / 2=S_{\text {lateral }}^{(n)} \sigma_{z}^{(n)}
$$

These compliances are inferred from suitable regions of linear dependence on the unloading path of the first cycle using linear regression.

Given the number of subcores tested, $N=6$, we only have 12 measurements, which poses a restriction on the degree of anisotropy that can be recovered. This is exacerbated by the fact that the orientations of subcores could not be related to the symmetry axes of anisotropy. In this situation, one has to resort to the use of additional external information and assumptions. In the case under study, the help comes from the fact that the rock is foliated with the known orientation of the foliation plane. One can therefore assume that the rock is approximately transverse-isotropic with the isotropy plane coinciding with the foliation plane. We shall use this assumption later on for the actual determination of the moduli.

In order to determine the set of anisotropic moduli corresponding to a certain, laboratory co-ordinate set, $(x$, $y, z)$ one needs to relate them to these two compliances measured in different subcores, and then use linear regression. This will be done using the six-dimensional matrix notation, as explained in the following subsections. 


\subsection{Matrix expression for anisotropic moduli and co-ordinate rotation}

Hereafter we refer to the anisotropic Hookes' law expressed in the following matrix notation (e.g. Lekhnitskii, 1977), where stress and strain are represented as 6 dimensional vectors and the tensor of elastic moduli or compliances as a 6 by 6 symmetrical matrix as follows:

$$
\left(\begin{array}{l}
\varepsilon_{11} \\
\varepsilon_{22} \\
\varepsilon_{33} \\
\varepsilon_{23} \\
\varepsilon_{13} \\
\varepsilon_{12}
\end{array}\right)=\left(\begin{array}{llllll}
S_{11} & S_{12} & S_{13} & S_{14} & S_{15} & S_{16} \\
S_{12} & S_{22} & S_{23} & S_{24} & S_{25} & S_{26} \\
S_{13} & S_{23} & S_{33} & S_{34} & S_{35} & S_{36} \\
S_{14} & S_{24} & S_{34} & S_{44} & S_{45} & S_{46} \\
S_{15} & S_{25} & S_{35} & S_{45} & S_{55} & S_{56} \\
S_{16} & S_{26} & S_{36} & S_{46} & S_{56} & S_{66}
\end{array}\right)\left(\begin{array}{l}
\sigma_{11} \\
\sigma_{22} \\
\sigma_{33} \\
\sigma_{23} \\
\sigma_{13} \\
\sigma_{12}
\end{array}\right), \text { or } \boldsymbol{\varepsilon}=\mathbf{S} \boldsymbol{\sigma}
$$

Similar notation is used to express stress versus strain through the matrix of elastic moduli $\mathbf{C}$.

We now discuss how the components of this representation change with co-ordinate rotations. In the usual $3 \mathrm{D}$ representation the co-ordinate rotation is specified by a $3 \times 3$ rotation matrix $\mathbf{R}$. The first row of the rotation matrix consists of the projections of a unit vector directed along the $x$-axis of the rotated co-ordinate set with respect to the original co-ordinate set; the second and the third rows of the matrix result from the projections of a unit vector directed along the $y$ - and $z$-axes, respectively. For our purposes it is convenient to express the general rotation as a superposition of (generally) 3 rotations with respect to $x, y$ and $z$ axes (see Figure 2):

$$
\mathbf{S}^{\prime}=\mathbf{Q S} \mathbf{Q}^{T}
$$

$$
\mathbf{Q}=\left(\begin{array}{cccccc}
R_{11}{ }^{2} & R_{12}{ }^{2} & R_{13}{ }^{2} & R_{12} R_{13} & R_{13} R_{11} & R_{12} R_{11} \\
R_{21}{ }^{2} & R_{22}{ }^{2} & R_{23}{ }^{2} & R_{23} R_{22} & R_{23} R_{21} & R_{22} R_{21} \\
R_{31}{ }^{2} & R_{32}{ }^{2} & R_{33}{ }^{2} & R_{33} R_{32} & R_{23} R_{31} & R_{32} R_{31} \\
2 R_{31} R_{21} & 2 R_{32} R_{22} & 2 R_{33} R_{23} & R_{33} R_{22}+R_{32} R_{23} & R_{33} R_{21}+R_{31} R_{23} & R_{31} R_{22}+R_{32} R_{21} \\
2 R_{31} R_{11} & 2 R_{32} R_{12} & 2 R_{33} R_{13} & R_{33} R_{12}+R_{32} R_{13} & R_{33} R_{11}+R_{31} R_{13} & R_{31} R_{12}+R_{32} R_{11} \\
2 R_{21} R_{11} & 2 R_{12} R_{22} & 2 R_{13} R_{23} & R_{13} R_{22}+R_{12} R_{23} & R_{13} R_{21}+R_{11} R_{23} & R_{11} R_{22}+R_{12} R_{21}
\end{array}\right)
$$

The transformation of the $6 \times 6$ compliance matrix $\mathbf{S}$ with the co-ordinate rotation is expressed as follows (e.g. Lekhnitskii, 1977):

$$
\mathbf{S}^{\prime}=\mathbf{Q S Q}^{T}
$$

$$
\mathbf{Q}=\left(\begin{array}{cccccc}
R_{11}{ }^{2} & R_{12}{ }^{2} & R_{13}{ }^{2} & R_{12} R_{13} & R_{13} R_{11} & R_{12} R_{11} \\
R_{21}{ }^{2} & R_{22}{ }^{2} & R_{23}{ }^{2} & R_{23} R_{22} & R_{23} R_{21} & R_{22} R_{21} \\
R_{31}{ }^{2} & R_{32}{ }^{2} & R_{33}{ }^{2} & R_{33} R_{32} & R_{23} R_{31} & R_{32} R_{31} \\
2 R_{31} R_{21} & 2 R_{32} R_{22} & 2 R_{33} R_{23} & R_{33} R_{22}+R_{32} R_{23} & R_{33} R_{21}+R_{31} R_{23} & R_{31} R_{22}+R_{32} R_{21} \\
2 R_{31} R_{11} & 2 R_{32} R_{12} & 2 R_{33} R_{13} & R_{33} R_{12}+R_{32} R_{13} & R_{33} R_{11}+R_{31} R_{13} & R_{31} R_{12}+R_{32} R_{11} \\
2 R_{21} R_{11} & 2 R_{12} R_{22} & 2 R_{13} R_{23} & R_{13} R_{22}+R_{12} R_{23} & R_{13} R_{21}+R_{11} R_{23} & R_{11} R_{22}+R_{12} R_{21}
\end{array}\right)
$$

where $R_{i j}$ are the entries of the 3D rotation matrix $\mathbf{R}$ and the superscript 'T' stands for matrix transposition. 


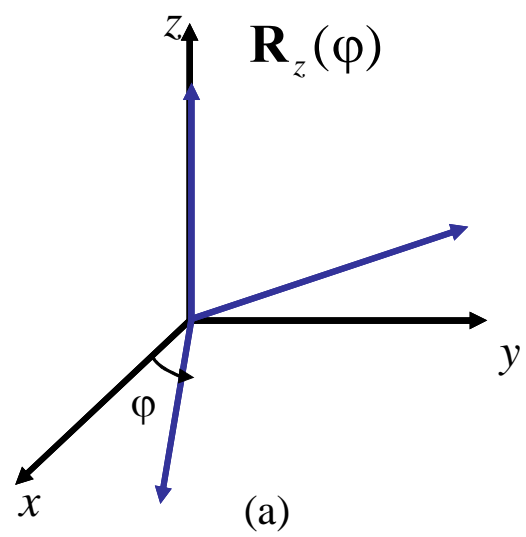

(a)

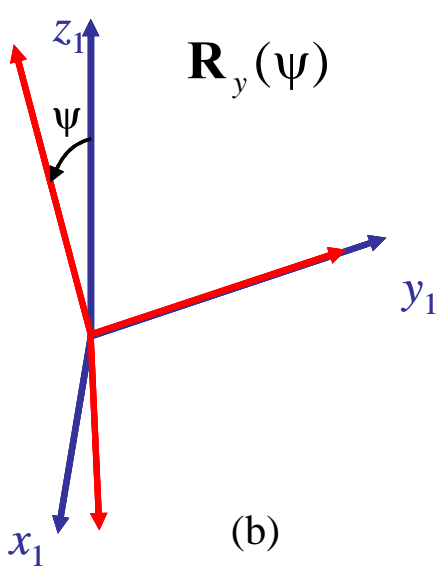

(b)

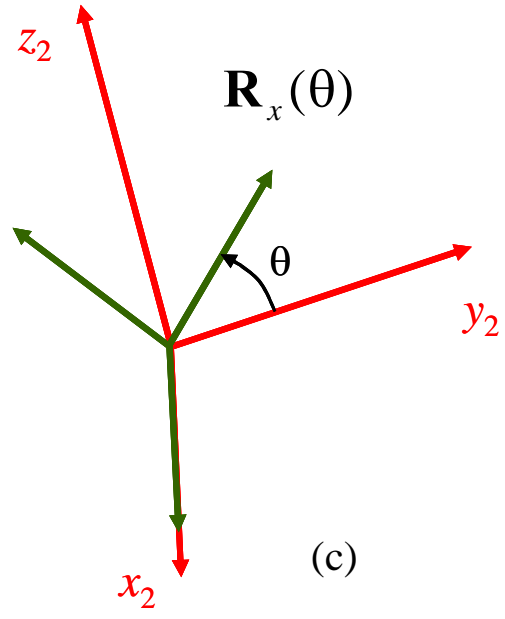

Figure 2 Three basic rotations: (a) rotation with respect to the z-axis, (b) rotation with respect to the $y$-axis, (c) rotation with respect to the $x$-axis

\subsection{Linear regression method of moduli reconstruction}

The general statement of the problem of recovering the anisotropic moduli or compliances from the data obtained from $N$ differently oriented subcores reads as follows: determine 21 independent components of the symmetric matrix $\mathbf{S}$ such that in the co-ordinate sets with the $z$-axes coinciding with the axis of the subcores, components $S_{33}$ and $\left(S_{13}+S_{23}\right) / 2$ are made close to the values of $S_{\text {axial }}^{(n)}$ and $S_{\text {lateral }}^{(n)}$ in the sense of the least sum of squares:

$$
\sum_{n=1}^{N}\left[\left(\frac{S_{13}^{(n)}+S_{12}^{(n)}}{2}-S_{\text {lateral }}^{(n)}\right)^{2}+\left(S_{33}^{(n)}-S_{\text {axial }}^{(n)}\right)^{2}\right] \rightarrow \text { min, where } \quad \mathbf{S}^{(n)}=\mathbf{Q}^{(n)} \mathbf{S} \mathbf{Q}^{(n)^{T}}
$$

Here $\mathbf{Q}^{(n)}$ is the rotation matrix that transfers the laboratory co-ordinate set to the co-ordinate set related to the $n$-th subcore.

Since the components of the compliance matrix represented in the direction of the $n$-th core linearly depend upon the unknown original components of the compliance matrix, the minimisation problem (5) can be reduced to multiple linear regressions. The simplest way of doing this is by introducing the following 21 axillary (basis) matrices $\mathbf{B}^{k m}$ specified by:

$$
B_{i j}^{k k}=\left\{\begin{array}{cc}
1 & \text { if } i=j=k \\
0 & \text { otherwise }
\end{array}, \quad B_{i j}^{k l}=\left\{\begin{array}{cc}
1 & \text { if } i=k, j=l \text { or } i=l, j=k \\
0 & \text { otherwise }
\end{array}\right.\right.
$$

We also need to extract the components of matrix $\mathbf{S}$ that correspond to (1). In order to accomplish this we introduce the following matrices:

$$
\mathbf{I}_{3}=\left(\begin{array}{l}
0 \\
0 \\
1 \\
0 \\
0 \\
0
\end{array}\right), \quad \mathbf{L}=\left(\begin{array}{llllll}
0 & 0 & \frac{1}{2} & 0 & 0 & 0 \\
0 & \frac{1}{2} & \frac{1}{2} & 0 & 0 & 0
\end{array}\right)
$$

such that: 


$$
\mathbf{L S I}_{3}=\left(\begin{array}{c}
S_{33} \\
\left(S_{13}+S_{23}\right) / 2
\end{array}\right)
$$

The least squares method (6) can now be expressed in the following matrix form:

$$
\sum_{\substack{k, l=1 \\
k<l}}^{6}\left|\mathbf{L} \mathbf{Q}^{(n)} \mathbf{B}^{k l} \mathbf{Q}^{(n)^{T}} \mathbf{I}_{3} S_{k l}-\mathbf{S}_{\exp }^{(n)}\right|^{2} \rightarrow \min , \quad \text { where } \quad \mathbf{S}_{\exp }^{(n)}=\left(\begin{array}{c}
S_{\text {axial }}^{(n)} \\
S_{\text {lateral }}^{(n)}
\end{array}\right), \quad n=1, \ldots, N
$$

where |.| denotes the vector length.

\subsection{Reconstruction of transverse-isotropic moduli}

As mentioned, we assume that the rock is transverse-isotropic with the isotropy plane coinciding with the foliation plane. Let $\mathbf{Q}^{(n)}$ be the rotation matrix that transfers the laboratory co-ordinate set to the co-ordinate set related to the $n$-th subcore, and $\mathbf{Q}^{\text {fol }}$ be the rotation matrix that rotates the laboratory set in such a way that its $z$-axis becomes normal to the foliation plane.

With respect to the co-ordinate set associated with the foliation plane the compliance matrix can be expressed through the transverse-isotropic moduli as follows (e.g. Lekhnitskii, 1977).

$$
\mathbf{S}=\left(\begin{array}{ccccccc}
S_{11} & S_{12} & S_{13} & 0 & 0 & 0 \\
S_{12} & S_{11} & S_{13} & 0 & 0 & 0 \\
S_{13} & S_{13} & S_{33} & 0 & 0 & 0 \\
0 & 0 & 0 & S_{44} / 2 & 0 & 0 \\
0 & 0 & 0 & 0 & S_{44} / 2 & 0 \\
0 & 0 & 0 & 0 & 0 & S_{11}-S_{12}
\end{array}\right)=\left(\begin{array}{cccccc}
\frac{1}{E} & \frac{-v}{E} & \frac{-v^{\prime}}{E^{\prime}} & 0 & 0 & 0 \\
\frac{-v}{E} & \frac{1}{E} & \frac{-v^{\prime}}{E^{\prime}} & 0 & 0 & 0 \\
\frac{-v^{\prime}}{E^{\prime}} & \frac{-v^{\prime}}{E^{\prime}} & \frac{1}{E^{\prime}} & 0 & 0 & 0 \\
0 & 0 & 0 & \frac{1}{2 G^{\prime}} & 0 & 0 \\
0 & 0 & 0 & 0 & \frac{1}{2 G^{\prime}} & 0 \\
0 & 0 & 0 & 0 & 0 & \frac{1+v}{E}
\end{array}\right)
$$

where $E, v$ are the Young's modulus and Poisson's ratio, respectively, corresponding to the loads in the foliation plane; and, $E^{\prime}, v^{\prime}$ and $G^{\prime}$ are the Young's modulus, Poisson's ratio and the shear modulus, respectively, corresponding to the loads normal to the foliation plane.

For this particular case it is convenient to use the single-index notation for the basis matrices (7). We have:

$$
\begin{aligned}
& \sum_{k=1}^{5}\left|\mathbf{B}_{\mathrm{k}}^{(n)} S_{k}-\mathbf{S}_{\exp }^{(n)}\right|^{2} \rightarrow \min \\
& \text { where } \quad \mathbf{B}_{k}^{(n)}=\mathbf{L} \mathbf{Q}^{(n)} \mathbf{Q}^{\text {fol }} \mathbf{B}^{k}\left(\mathbf{Q}^{(n)} \mathbf{Q}^{\text {fol }}{ }^{-1}\right)^{T} \mathbf{I}_{3}, \quad \mathbf{S}_{\exp }^{(n)}=\left(\begin{array}{c}
S_{\text {axial }}^{(n)} \\
S_{\text {lateral }}^{(n)}
\end{array}\right), \quad n=1, \ldots, N
\end{aligned}
$$

The least squares method (10) can now be expressed as: 


$$
\begin{aligned}
& \sum_{k=1}^{5}\left|\mathbf{B}_{\mathrm{k}}^{(n)} S_{k}-\mathbf{S}_{\exp }^{(n)}\right|^{2} \rightarrow \min \\
& \text { where } \quad \mathbf{B}_{k}^{(n)}=\mathbf{L} \mathbf{Q}^{(n)} \mathbf{Q}^{\text {fol }} \mathbf{B}^{k}\left(\mathbf{Q}^{(n)} \mathbf{Q}^{f o l^{-1}}\right)^{T} \mathbf{I}_{3}, \quad \mathbf{S}_{\exp }^{(n)}=\left(\begin{array}{c}
S_{\text {axial }}^{(n)} \\
S_{\text {lateral }}^{(n)}
\end{array}\right), \quad n=1, \ldots, N
\end{aligned}
$$

Here the basic compliances are $S_{1}=S_{11}, S_{2}=S_{33}, S_{3}=S_{12}, S_{4}=S_{13}, S_{5}=S_{44}$.

The problem (13) can be solved by introducing a matrix $\mathbf{X}$ consisting of $2 N$ rows and 5 columns, the $k$-th column being formed by concatenation of $N$ vectors $\mathbf{B}_{k}^{(n)}$. Then the vector of the basic compliances $\mathbf{S}^{b}=\left(S_{k}\right), k=1, \ldots, 5$ can be obtained as (e.g. Hakala et al., 2007).

$$
\mathbf{S}^{b}=\left(\mathbf{X}^{T} \mathbf{X}\right)^{-1} \mathbf{X}^{T} \mathbf{Y}
$$

where the column-vector of measurements $\mathbf{Y}$ consists of $2 N$ rows formed by concatenation of $N$ vectors $\mathbf{S}_{\exp }^{(n)}$.

\subsection{Results}

In the laboratory tests considered, the orientations of the subcores and the values of local compliances are given in Table 1.

Table 1 Results of 6 laboratory measurements. The compliances are in $\mathrm{MPa}^{-1}$

\begin{tabular}{cccc} 
Azimuth/Trend & Plunge & $\begin{array}{c}\text { Axial } \\
\text { Compliance }\end{array}$ & $\begin{array}{c}\text { Lateral } \\
\text { Compliance }\end{array}$ \\
\hline $47^{\circ}$ & $5^{\circ}$ & $1.42213 \mathrm{E}-05$ & $-5.482 \mathrm{E}-06$ \\
$227^{\circ}$ & $85^{\circ}$ & $1.57651 \mathrm{E}-05$ & $-3.533 \mathrm{E}-06$ \\
$137^{\circ}$ & $0^{\circ}$ & $1.54532 \mathrm{E}-05$ & $-4.295 \mathrm{E}-06$ \\
$142^{\circ}$ & $45^{\circ}$ & $1.39184 \mathrm{E}-05$ & $-3.143 \mathrm{E}-06$ \\
$47^{\circ}$ & $50^{\circ}$ & $1.29886 \mathrm{E}-05$ & $-2.999 \mathrm{E}-06$ \\
$2^{\circ}$ & $4^{\circ}$ & $2.48136 \mathrm{E}-05$ & $-5.766 \mathrm{E}-06$ \\
\hline
\end{tabular}

In order to relate the subcore orientations given in azimuth and plunge to the angles of basic rotations (4), we direct the $y$-axis of the laboratory co-ordinate set towards the North and the $z$-axis upwards. Then $\mathbf{Q}^{(n)}$ can be obtained from the rotation matrix $\mathbf{R}_{z}$ (-azimuth) $\mathbf{R}_{x}$ (plunge- $90^{\circ}$ ) using the representation (4). The foliation is given by azimuth $=96^{\circ}$ and plunge $=72^{\circ}$. This corresponds to the rotation matrix $\mathbf{R}_{z}\left(-96^{\circ}\right) \mathbf{R}_{x}\left(72^{\circ}-180^{\circ}\right)$, which can be converted into $\mathbf{Q}^{\text {fol }}$ by using (5).

After substituting these matrices into (13), (14) one obtains the following values for transverse-isotropic compliances (in $\mathrm{MPa}^{-1}$ ):

$$
S_{11}=2.06 * 10^{-5}, S_{33}=2.12 * 10^{-5}, S_{12}=-0.472 * 10^{-5}, S_{13}=0.777 * 10^{-5}, S_{44}=4.43 * 10^{-5}
$$

and moduli:

$$
E=48.5 G P a, \quad E^{\prime}=47.1 G P a, \quad v=0.23, \quad v^{\prime}=0.37, \quad G^{\prime}=29.1 G P a
$$

It is seen that the Young's moduli do not differ much. The main indicator of anisotropy is the shear modulus $G$ ' corresponding to the direction normal to the isotropy plane: in the isotropic case it should be $19.7 \mathrm{GPa}$, 
which is around $50 \%$ smaller than $G^{\prime}$. Another indicator of anisotropy is the difference in the Poisson's ratio values.

\subsection{Sensitivity analysis}

The reconstruction of moduli is based on the results of laboratory tests, which are usually accompanied by random errors coming from different sources that are beyond the control of the lab technicians. These errors are usually not large, but if the reconstruction procedure is sensitive to errors it might magnify them to a level obstructing the actual values of the moduli. In order to check this possibility we conducted sensitivity analyses based on computer simulations of moduli reconstruction from the randomly disturbed results of the measurements. We assumed the uniform and independent disturbance of the measurements without systematic errors. The interval of disturbance was $\pm 10 \%$, which gives the value of coefficient of variation (ratio between the standard deviation and the mean value) of 5.8\%. Table 2 shows the resulting mean values and the coefficient of variation obtained after 1000 simulations. It is seen that the mean values correspond quite well to the moduli reconstructed from the undisturbed data, however the coefficients of variations can be quite high suggesting a potential for sensitivity of the reconstruction procedure.

Two ways of improving the procedure can be suggested. Firstly, the fact that the mean values give a very high accuracy suggests that simply increasing the number of measurements will ensure accurate moduli reconstruction. Secondly, we note that the reconstruction procedure is based on matrix $\mathbf{X}$, which is a function of the subcore directions. These directions are currently specified by the needs of the DRA analysis. Apparently, there is room for an optimisation problem: determine the subcore directions that minimise the sensitivity of the moduli reconstruction procedure. Given that the moduli reconstruction is conducted alongside the DRA testing, the latter will impose constraints on the minimisation problem, but only insofar as there is sufficient core for subsampling.

Table 2 Results of computer simulations of moduli reconstruction from randomly disturbed measurements without systematic error. The coefficient of variation of the disturbance is $5.8 \%$

\begin{tabular}{lccc}
\hline & $\begin{array}{c}\text { Moduli recovered from } \\
\text { original measurements }\end{array}$ & $\begin{array}{c}\text { Mean values of moduli obtained } \\
\text { from disturbed measurements }\end{array}$ & $\begin{array}{c}\text { The coefficients of } \\
\text { variation (\%) }\end{array}$ \\
\hline $\boldsymbol{E}$ & $48.5 \mathrm{GPa}$ & $48.7 \mathrm{GPa}$ & 3.6 \\
$\boldsymbol{E}^{\prime}$ & $47.1 \mathrm{GPa}$ & $50.1 \mathrm{GPa}$ & 33 \\
$\boldsymbol{v}$ & 0.23 & 0.23 & 56 \\
$\boldsymbol{v}^{\prime}$ & 0.37 & 0.36 & 16.5 \\
$\boldsymbol{G}$ & $29.1 \mathrm{GPa}$ & $29.1 \mathrm{GPa}$ & 6.5 \\
\hline
\end{tabular}

\section{DRA stress reconstruction results and comparisons}

The stress determination using the isotropic solution for the HI cell and the DRA approach are summarised in Table 3 and Figure 3. The results can only be considered similar in terms of the absolute magnitudes. In percentage terms there is considerable discrepancy. Clearly, this requires resolution as at this stage most credence in stress measurement is given to HI cell tests irrespective of the influence of anisotropy. 
Table 3 Test results for two measurement techniques

\begin{tabular}{llllccc}
\hline & \multicolumn{3}{c}{ HI Cell } & \multicolumn{3}{c}{ DRA } \\
\cline { 2 - 7 } & Bearing & Plunge & MPa & Bearing & Plunge & MPa \\
\cline { 2 - 7 }$\sigma_{1}$ & $206^{\circ}$ & $12^{\circ}$ & 13.4 & $11^{\circ}$ & $61^{\circ}$ & 11.5 \\
$\sigma_{2}$ & $116^{\circ}$ & $1^{\circ}$ & 9.6 & $266^{\circ}$ & $9^{\circ}$ & 10.3 \\
$\sigma_{3}$ & $23^{\circ}$ & $78^{\circ}$ & 7.2 & $171^{\circ}$ & $28^{\circ}$ & 3.5 \\
\hline
\end{tabular}

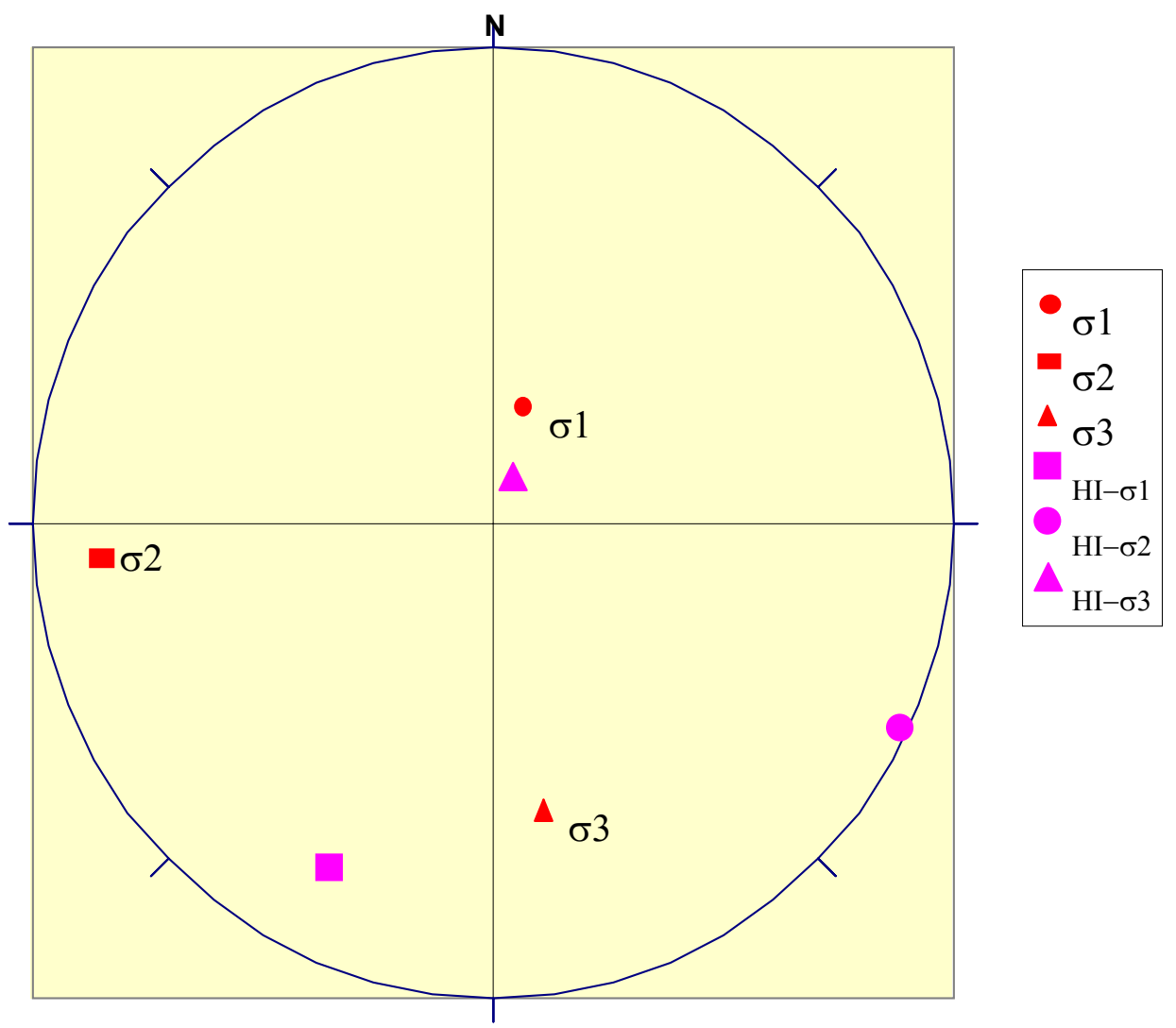

Figure 3 Stereonet of the stress determination - HI Isotropic solution (large symbols) versus DRA results

\section{Conclusion}

Comparison between the stresses recovered from the DRA analysis and the HI cell based on the assumption of isotropy of the rock showed quite a considerable difference, predominantly in identifying the principal directions. This suggests that the assumption of rock isotropy is oversimplifying. The rock is foliated and the associated anisotropy could considerably alter the results of HI cell reconstruction. The DRA analysis is insensitive to the elastic properties of the rock.

A by-product of the DRA testing is the determination of moduli in the directions coinciding with the subcore axes. We presented a general procedure for the reconstruction of anisotropic moduli from the laboratory tests of subcores oriented in a number of different directions. Generally, 21 components of the tensor of compliances should be recovered, which highlights the necessity to have quite a large number of measurements. Even if the degree of anisotropy is not that high and, as often happens in practice, orthotropy or even transverse isotropy can be assumed, the problem is complicated by the fact that the orientations of the symmetry planes or axis (in transverse-isotropic case) with respect to the subcores are not known. It is therefore paramount to be able to identify the rock symmetry independently using petrophysical and 
geological data. In particular, in the example considered a foliation plane was identifiable, which allowed us to assume that the rock is transverse isotropic with the isotropy plane coinciding with the foliation plane. In that case the proposed procedure leads to relatively simple and accurate reconstruction of the elastic moduli.

The procedure presented in this paper is moving to provide a basis for interpreting HI tests in anisotropic rock. The fabric in the rock is oblique to the cell and hence there is an apparent modulus change, but based on the transverse isotropic solution this is more likely to be a phenomenon associated with a variation in the Poisson's ratio and the shear modulus as noted in this paper. Further work is now required to resolve the analysis of the $\mathrm{HI}$ cell test in a transverse isotropic rock mass.

\section{References}

Amadei, B. (1996) Importance of anisotropy when estimating and measuring in-situ stress in rock. Int. J. Rock Mech. Min. Sci. Vol. 33(3), pp. 293-325.

Amadei, B. (1983) In: C.A. Brebbia and S.A. Orszag (eds) Lecture notes in engineering - rock anisotropy and theory of stress measurements. Springer Verlag, Berlin, pp. 233-41.

Amadei, B. and Goodman, R.E. (1982) The Influence of Rock Anisotropy on Stress Measurements by Overcoring Techniques, Rock Mechanics, Vol. 15, pp. 167-180.

Barr, S.P., Jupe, A.J. and Hunt, D.P. (1999) The Kaiser effect for samples pre-stressed at $820 \mathrm{~m}$ and $2.4 \mathrm{~km}$ with stress tensor results. Proceedings $9^{\text {th }}$ Int. Cong. Rock Mech. 28, pp. 1136-1143.

Dight, P.M. (2006) Determination of Insitu Stress from Oriented Core. Proceedings International Symposium on In-situ Rock Stress, Trondheim, Norway, pp. 167-175.

Goodman, R.E. (1963) Subaudible noise during compression of rocks. Bull. Geol. Soc. Am. Vol. 74, pp. 487-490.

Hakala, M., Kuula, H. and Hudson, J.A. (2007) Estimating the transversely isotropic elastic intact properties for in-situ stress measurement data reduction: A case study of the Olkiluoto mica gneiss, Finland. Int. Jnl. Rock Mechanics and Mining Sciences, Vol. 44, pp. 14-46.

Kaiser, J. (1950) An investigation into the occurrence of noises in tensile tests or a study of acoustic phenomena in tensile tests. PhD. Thesis. Tech Hochschule, Munich, Germany.

Kaiser, J. (1953) Erkenntnisse und Folgerungen aus der Messung von Gerauschen bei Zugbeanspruchung von Metallischen Werstoffen. Archiv Eisenhuttenwesen, Vol. 24, pp. 43-45.

Lavrov, A. (2002) The Kaiser effect in rocks: principles and stress estimation techniques. Int. Jnl. Rock Mech. Min. Sci. Vol. 40, pp. 151-171.

Kurita, K. and Fujii, N. (1979) Stress memory of crystalline rocks in acoustic emission. Geophys Res Lett. Vol. 6, pp. 9-12.

Leeman, E.R. (1965a) The measurement of stress in rock. Part I. The principles of rock stress measurements. Jnl of South African Institute of Mining and Metallurgy, pp. 248-284.

Leeman, E.R. (1965b) The measurement of stress in rock. Part II. Borehole rock stress measuring instruments. Jnl of South African Institute of Mining and Metallurgy, pp. 285-317.

Leeman, E.R. (1965c) The measurement of stress in rock. Part III. The results of some rock stress investigations. Jnl of South African Institute of Mining and Metallurgy, pp. 318-374.

Lekhnitskii, S.G. (1977) Theory of Elasticity of an Anisotropic Body. Mir, Moscow.

Lim, S.S., Martin C.D. and Christiansson, R. (2006) Estimating in-situ stress magnitudes from core disking. Proceedings International Symposium on In-situ Rock Stress, Trondheim, Norway, pp. 159-166.

Meyer, A.G., Hunt, S.P. and Oliver, K.J. (2002) The use of the DRA Technique and Porosimetry for Estimating the Maximum In-situ Stress in Rock from Core. Australian Geomechanics Journal, Vol. 37(1), pp. 17-22.

Seto, M. and Villaescusa, E. (1999) In-situ Stress Determination by Acoustic Emission Techniques from McArthur River Mine Cores. Proc $8^{\text {th }}$ Aust. NZ. Conf. Geomech, Hobart, Vol. 2, pp. 929-934.

Villaescusa, E., Windsor, C., Li, J., Baird, G. and Seto, M. (2003) Stress Measurements from cored rock. MERIWA Report M329.

Worotnicki, G. (1993) CSIRO triaxial stress measurement cell. In J.A. Hudson (editor), Comprehensive Rock Engineering, Vol. 3, Pergamon Press, Oxford, pp. 329-394.

Yamamoto, K., Kuwahara, Y., Kato, N. and Hirasawa, T. (1990) Deformation rate analysis: a new method for in-situ stress estimation from inelastic deformation of rock samples under uniaxial compression. Tohoku Geophysical Journal Vol. 33, pp. 1-13.

Yamamoto, K. (1995) The rock property of in-situ stress memory: Discussions on its mechanism. Int. Workshop on Rock Stress: Measurements at Great Depth. Matsuki and Sugawara (eds), 8 th $I S R M$, Tokyo, Sept 35-30, pp. 46-51.

Yamamoto, K., Yamamoto, H., Kato, N. and Hirasawa, T. (1991) Deformation Rate Analysis for In Situ Stress Estimation, $5^{\text {th }}$ Int. Conf. on Acoustic Emission/Microseismic Activity in Geologic Structures and Materials Pennsylvania State University, 11-13 June, pp. 243-255. 